Virappa Virupaxappa Pattanshetti*, G.M. Shashidhara and Mysore Guruswamy Veena

\title{
Dielectric and thermal properties of magnesium oxide/poly(aryl ether ketone) nanocomposites
}

https://doi.org/10.1515/secm-2016-0273

Received September 28, 2016; accepted July 14, 2017; previously published online October 14, 2017

\begin{abstract}
In the present study, dielectric and thermal properties of poly(aryl ether ketone) (PAEK)-nanocomposites with varying weight percentage of magnesium oxide $(\mathrm{MgO})$ have been reported. The thermal properties of PAEK/MgO nanocomposites were studied using differential scanning calorimetry, thermogravimetric analysis, thermo-mechanical analysis and thermal conductivity. Transmission electron microscopy of the developed nanocomposites shows agglomerate-free dispersion of $\mathrm{MgO}$ nanoparticles in PAEK matrix. From the dielectric properties, dielectric constant of 13 was achieved for $10 \mathrm{wt} \%$ $\mathrm{PAEK} / \mathrm{MgO}$ nanocomposite at $230^{\circ} \mathrm{C}$. Further, minimum $\tan \delta$ and maximum volume resistivity were found for 10 wt $\%$ PAEK/MgO nanocomposite. Data from thermal studies indicate that the incorporation of $\mathrm{MgO}$ into PAEK significantly enhanced the glass transition temperature and slightly deteriorated the thermal stability, char yield, and flame-retardant properties. Also, highest thermal conductivity and lowest coefficient of thermal expansion were achieved at $10 \mathrm{wt} \%$ of the $\mathrm{MgO}$ content. An excellent combination of both dielectric and thermal properties of the PAEK/MgO nanocomposites developed in the present study finds its potential application in microelectronics and electrical insulation in power equipments.
\end{abstract}

Keywords: dielectric constant; magnesium oxide; poly(aryl ether ketone) (PAEK) nanocomposites; thermal conductivity; thermal stability.

\footnotetext{
*Corresponding author: Virappa Virupaxappa Pattanshetti, Central Power Research Institute, Bengaluru, Karnataka, India; and JSS Research Foundation, JSS Technical Institutions Campus, Sri Jayachamarjendra College of Engineering, JSS S \&T University, Mysuru 570006, Karnataka, India, Phone: +91 9449836878 , Fax:+91 080 23604448, e-mail: virappa.pattanshetti@yahoo.com G.M. Shashidhara: JSS Research Foundation, JSSTIC, SJCE, Mysuru 570006, Karnataka, India; and Department of Polymer Science and Technology, Sri Jayachamarajendra College of Engineering, Mysuru 570006, Karnataka, India

Mysore Guruswamy Veena: Department of Electronics and Communication Engineering, Sri Jayachamarajendra College of Engineering, Mysuru 570006, Karnataka, India
}

\section{Introduction}

Power equipment such as transformers conventionally use oil and paper as a winding insulation. Insulation in these systems serves as an insulant and a coolant for a long duration of lifetime. It has been found that, over a period of time, the insulation becomes vulnerable for oxidation and degrades thereby limiting the life of the power equipment; problems are more severe in tropical countries due to high humidity and high temperature conditions [1]. Insulation winding, insulant and coolant are critical components in the power system network and their performance will have an impact on the power system reliability. In this study we have selected a high-grade thermoplastic engineering material poly(aryl ether ketone) (PAEK) as the thermoplastic polymer matrix representing a class of interest for both scientific research and application at an industrial level and a high-temperature insulant magnesium oxide ( $\mathrm{MgO})$ nanoparticles for reinforcement.

Commercially, PAEK is considered as a high-performance engineering thermoplastic material with an excellent combination of chemical, physical and mechanical properties which makes them a class of important advanced materials used in chemical, medical and automotive industries. Conventional PAEK shows high crystalline properties, very high melting temperature and poor solubility towards organic solvents, restricting their application to thin films and coating materials. The three series of modified PAEK/metallophthalocyanine composites were prepared by $\mathrm{Xu}$ et al. [2] using the solution blending technique. The spherical shape of the prepared composites results in better interphase interactions with metallophthalocyanine and the composites showed better dielectric properties with a dielectric constant $>400$ and a dielectric loss of $<0.5$ at a frequency of $100 \mathrm{~Hz}$. The PAEKs that were derived from bisphenol monomers using two-step synthesis [3] showed excellent crystallinity, thermal, mechanical and electrical properties. The preparation of high-performance PAEK/ZnO nanocomposites was carried out using planetary ball mill followed by hot pressing [4]. There was a significant increase in the dielectric constant and the thermal stability of nanocomposites with an increase in the content of $\mathrm{ZnO}$ nanoparticles in the PAEK matrix. The influence of 
the incorporation of polyhedral oligomeric silsesquioxane particles on fluoropoly(ether ether ketone) s (PEEK$\mathrm{CF}_{3}-\mathrm{COOH}$ ) nanocomposites has been investigated [5]. The dielectric constants of the organic-inorganic nanocomposites were drastically reduced relative to neat PEEK$\mathrm{CF}_{3}-\mathrm{COOH}$ films. The thermal and mechanical properties of these nanocomposites were significantly improved by the incorporation of polyhedral oligomeric silsesquioxane moieties. Phenyltrimethoxysilane-treated $\mathrm{SiO}_{2}$ nanoparticles filled poly(ether ether ketone) (PEEK) matrix nanocomposites were prepared using hot pressing [6]. The result shows that the prepared nanocomposites exhibited lower coefficient of thermal expansion (CTE), lower melting point, good thermal stability and glass transition temperature due to the presence of $\mathrm{SiO}_{2}$ nanoparticles. There was a slight reduction in the dielectric constant and $\tan \delta$ value for untreated nanocomposites.

The characterization of PEEK nanocomposites containing nano-sized aluminum oxide $\left(\mathrm{Al}_{2} \mathrm{O}_{3}\right)$ filler up to $30 \mathrm{wt} \%$ loading exhibits improved thermal stability, crystallization and CTE $[7,8]$. The synthesis and characterization of proton-conducting composites of sulfonated PEEK with organically functionalized titanium dioxide $\left(\mathrm{TiO}_{2}\right)$ nanoparticles present in inhomogeneous structure [9] showed high strength and low ductility, high water uptake and proton-conductivity as compared to hydrophobic $\mathrm{TiO}_{2}$ with homogeneous structure. The authors fine-tuned the surface treatment of nanoparticles by compromising with the high conductivity and homogeneous structure resulting in proton exchange membrane fuel cells [9]. For better dispersion of nanozirconia in PEEK matrix and PEEK/ zirconia nanocomposites a method of powder mixing followed by melt blending process was carried out [10]. The PEEK/zirconia nanocomposites showed higher thermal stability than pure PEEK and there was no change in $T_{\mathrm{g}}$. Based on enhanced dispersion, some of the mechanical properties of nanocomposites have been increased drastically. An elevated heat distortion temperature and improved dynamic mechanical properties were observed for the PAEK/clay nanocomposites [11]. The composite properties were dependent on the content and modifier structures of the organoclay. The results showed that, ball milling pristine clay improved the composite properties due to homogeneous dispersion to a certain extent, similar to the modified clay.

Worldwide, many researchers are working towards improving the chemical, physical and mechanical properties of PAEK, but the available literature for the study of electrical and dielectric properties with temperature variation is very scarce. The novelty of this work is based on the fabrication of PAEK reinforced with $\mathrm{MgO}$ nanoparticles prepared by a sol-gel process, up to $10 \mathrm{wt} \%$ loading which can find its application in power systems. With respect to the insulation the high capacity power transformer should function as a good dielectric and dissipate the heat generated. The resistive heating of the windings leads to a high temperature in the power transformer which has to be instantaneously cooled, otherwise the high temperature may cause transformer failure. If PAEK/MgO nanocomposites are used as insulators for power transformer application, the improvement in the thermal conductivity and the corresponding heat capacity can be observed. The nanocomposite may act as a heat sink which dissipates the reaction heat and prevents the local overheating of the material resulting in the reduction of shrinkage stresses. As the insulation winding, insulant and coolant are critical components in the power system network the satisfactory performance of these will have an impact on the power system's reliability. Hence, the present research work focuses on the development of high-performance PAEK/MgO polymer nanocomposites as a promising material for the next generation insulating system in enhancing the life of power equipment components and thereby ensuring the power system's reliability.

\section{Experimental and measurements}

\subsection{Materials and methods}

The commercial PAEK, (grade G-PAEK 1200 P) in powder form was purchased from M/s Gharda chemicals, Mumbai, India. The G-PAEK $1200 \mathrm{P}$ was a gray color powder with a melting point of $372^{\circ} \mathrm{C}$, density of $1.3 \mathrm{~g} / \mathrm{cm}^{3}$, and crystallinity of $>40 \%$. Magnesium oxide $(\mathrm{MgO})$ nanofiller was prepared and developed in-house by the sol-gel process. The gel consisting of the solution of magnesium nitrate and urea was prepared in an aqueous system in a ratio of 1:5. In this gel network magnesium nitrate species was uniformly surrounded by the fuel molecules. Then this gel matrix was heated at a temperature of $550^{\circ} \mathrm{C}$ as the microexplosions of species take place leading to the formation of nanometric metal oxide particles. The stabilized gels consist of highly porous metal oxide matrix, upon grinding and dispersion it produces nano-sized metal oxide fillers having dimensions of $25-35 \mathrm{~nm}$ and having a density of $3.58 \mathrm{~g} / \mathrm{cm}^{3}$.

\subsection{Nanocomposite fabrication}

The procedure for the preparation of pure PAEK and PAEK/ MgO composite is explained as below: 
To obtain pure PAEK polymer matrix, the PAEK powder was initially dried for $4 \mathrm{~h}$ in an oven maintained at $150^{\circ} \mathrm{C}$. Then $1000 \mathrm{~g}$ of PAEK was weighed and fed to the twin screw extruder (Omega 20 Steer Engineering, Bengaluru make) by maintaining temperature at eight different zones from $340^{\circ} \mathrm{C}$ to $395^{\circ} \mathrm{C}$ to bring the material into molten state. The extrudate from the extruder was air cooled, dried and passed through a pelletizer.

To obtain $2 \mathrm{wt} \%$ of PAEK/MgO nanocomposite $980 \mathrm{~g}$ of pre-dried G-PAEK powder and $20 \mathrm{~g}$ of $\mathrm{MgO}$ nanopowder suspended in $300 \mathrm{ml}$ acetone were taken ( $\mathrm{MgO}$ suspension). In the first step MgO suspension and only $200 \mathrm{~g}$ of dried G- PAEK powder was manually mixed using mortar and pestle, and dried to remove the solvent. This resultant mixture together with the remaining $780 \mathrm{~g}$ G-PAEK powder is thoroughly mixed in an industrial dry mixer. Finally, this mixture was fed to a twin screw extruder for material to get molten and mixed well by maintaining temperature at eight different zones from $340^{\circ} \mathrm{C}$ to $395^{\circ} \mathrm{C}$. The extrudate from the extruder was air cooled, dried and passed through a pelletizer.

Similarly, for $4 \mathrm{wt} \%$ of PAEK/MgO nanocomposite $960 \mathrm{~g}$ of dried G-PAEK powder and mixed $40 \mathrm{~g}$ of $\mathrm{MgO}$ nanopowder suspended in $300 \mathrm{ml}$ acetone were taken. The suspension was mixed with $200 \mathrm{~g}$ of pre-dried G-PAEK powder. The mixture was mixed with mortar and pestle and dried to remove the solvent. The mixture was then mixed with the remaining amount of G-PAEK powder, i.e. $760 \mathrm{~g}$. For $6 \mathrm{wt} \%$ of PAEK/MgO nanocomposite $940 \mathrm{~g}$ of G-PAEK powder and $60 \mathrm{~g}$ of $\mathrm{MgO}$ powder and for $10 \mathrm{wt} \%$ of PAEK/MgO nanocomposite $900 \mathrm{~g}$ of G-PAEK powder and $100 \mathrm{~g}$ of $\mathrm{MgO}$ powder were appropriately added to obtain the pellets. Similar sub-mixture procedure was used, i.e. $\mathrm{MgO}$ powder suspended in acetone was mixed with 200 $\mathrm{g}$ of G-PAEK, dried to remove the solvent and then mixed with the remaining G-PAEK powder. The PAEK/MgO nanocomposite pellets obtained by this process were fed into the injection molding machine maintained at a temperature of $385^{\circ} \mathrm{C}$ (Mallik Engineering, Mumbai, India). Then the pellets were ejected from the mold cavity. The size of the molded disc was about $110 \mathrm{~mm}$ diameter $\times 3 \mathrm{~mm}$ thick. These discs were finished by abrading with emery papers, cleaned with acetone and dried. Finally, they are used for the characterization of the dielectric and thermal properties.

\subsection{Morphological studies}

Morphological analysis of PAEK and PAEK/MgO nanocomposites was conducted using Philips XL 20 scanning electron microscope (SEM). For SEM of nanocomposites, a small piece of the sample was cut from the disc specimen manually, polished with successively finer grades of emery paper followed by a cloth to remove scratches. The samples were coated with a thin layer of gold to make the sample electrically conducting. The dispersion of $\mathrm{MgO}$ nanoparticles in PAEK matrix was also observed using transmission electron microscopy (TEM) (Joel Ltd., Tokyo, Japan) 100 CXII system with a standard magnification of $360-320,000 \times$.

\subsection{Electrical measurements}

The volume resistivity $\left(\rho_{\mathrm{v}}\right)$ measurement was performed using an Eltel-Automatic Resistivity Test Set kit typeADTR-2K (EL TEL, Bengaluru, India) and tetex bridge was used to measure the capacitance $(C)$ and dissipation factor $(\tan \delta)$ over a temperature range of $30^{\circ} \mathrm{C}-230^{\circ} \mathrm{C}$. From the measured capacitance, dielectric constant $\left(\varepsilon_{\mathrm{r}}\right)$ is calculated for each sample. These experiments were carried out according to ASTM D 257 and ASTM D 150, respectively.

\subsection{Differential scanning calorimetry}

The effect of $\mathrm{MgO}$ nanoparticles on the melting characteristics of PAEK was measured using differential scanning calorimetry (TA Instruments, DSC model 821, USA) operated under a nitrogen atmosphere at a heating rate of $10^{\circ} \mathrm{C} / \mathrm{min}$ in the temperature range of $50^{\circ} \mathrm{C}-400^{\circ} \mathrm{C}$.

\subsection{Thermo-mechanical analysis}

Thermo-mechanical analyzer (TMA) (TA Instruments, TA450, USA) was used to assess the penetration and dimensional changes of PAEK/MgO nanocomposites over a temperature range of $25^{\circ} \mathrm{C}-350^{\circ} \mathrm{C}$. It consists of a highly sensitive linear variable differential transformer, low mass furnace and a precise motor for probe loading. Samples were run at a heating rate of $10^{\circ} \mathrm{C} / \mathrm{min}$ from room temperature to $350^{\circ} \mathrm{C}$ under nitrogen atmosphere. The experiments were carried out in accordance with ASTM E 1131.

Thermal conductivity studies were carried out by the hot transient method as per ASTM 22007 Part 2, 2008 using TPS 500 model thermal conductivity analyzer. The penetration probe and general probe were used for TMA evaluation of PAEK/MgO nanocomposites. The area of penetration was calculated by triangle approximation. The penetration probe has a contact diameter of $0.89 \mathrm{~mm}$ 
and the expansion probe has a diameter of $2.54 \mathrm{~mm}$. The probe was subjected to a load of $0.5 \mathrm{~N}$ and the experiment was carried out as per ISO 1359 Part 3.

The CTE behavior of PAEK nanocomposites was carried out using the expansion probe method (TA instruments Q400 make, USA). The diameter of expansion probe was $3 \mathrm{~mm}$ and the measurements were made at an applied force of $0.5 \mathrm{~N}$ under nitrogen atmosphere with a flow of $50 \mathrm{ml} / \mathrm{min}$. The dimensional change with temperature $\left(\mu \mathrm{m} /{ }^{\circ} \mathrm{C}\right)$ was measured for all the nanocomposites.

\section{Results and discussion}

\subsection{Dispersion of $\mathrm{MgO}$ nanoparticles in PAEK}

The dispersion of $\mathrm{MgO}$ nanofiller in PAEK polymer was observed using TEM and the image is shown in Figure 1. The TEM image of the PAEK/MgO nanocomposites showed that the particles are in the nanometric dimensions of $\sim 35 \mathrm{~nm}$. The metal nitrate and the urea solution were allowed for more than $48 \mathrm{~h}$ which enhances the nanoparticle size range and its distribution. The uniformity of the distribution of $\mathrm{MgO}$ nanoparticles in PAEK matrix polymer was examined by SEM images. Figure $2(\mathrm{~A}-\mathrm{D})$ represents the SEM micrographs of 0, 2, 6 and $10 \mathrm{wt} \% \mathrm{MgO}$-filled PAEK nanocomposites, respectively. These micrographs show a homogenous morphology. No localized domains and no phase segregation were observed even at the nanometer scale, implying that $\mathrm{MgO}$ nanoparticles were well dispersed in the PAEK matrix without any MgO aggregation.

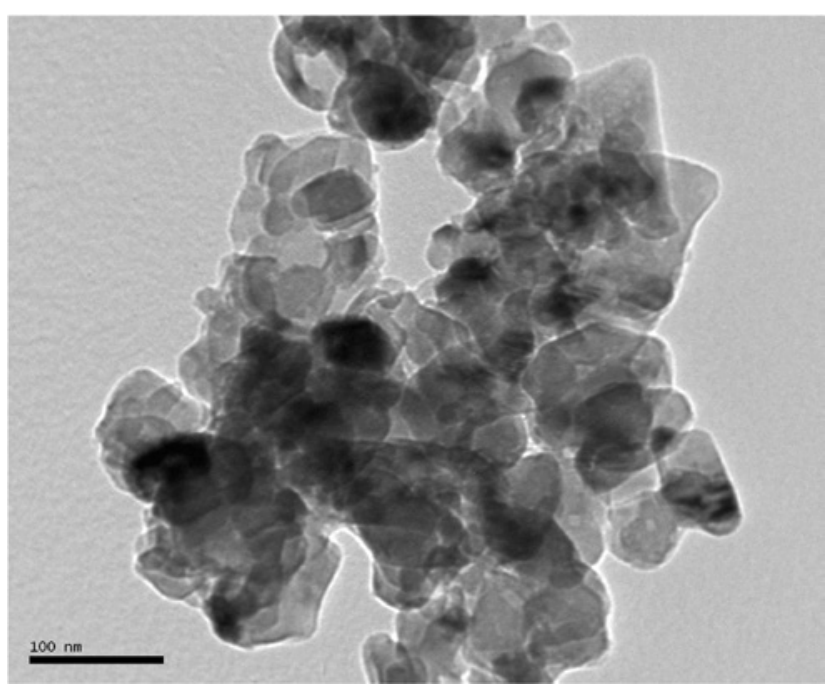

Figure 1: TEM image of MgO nanoparticles.
In the present study the experiments are carried out for the fabrication of unmodified $\mathrm{MgO}$ nanoparticles in PAEK matrix. In future work, modification of $\mathrm{MgO}$ nanoparticles is carried out and the results are compared.

\subsection{Dielectric properties}

The dielectric property of the material generally depends on the mechanism of interaction of the molecules with the applied electric field. Figure 3 shows the effect of frequency on dielectric constant $\left(\varepsilon_{\mathrm{r}}\right)$ of the PAEK/MgO nanocomposites.

\subsubsection{Effect of frequency on dielectric constant}

The variations of dielectric constant $\left(\varepsilon_{\mathrm{r}}\right)$ for different frequencies of PAEK/MgO nanocomposites with $\mathrm{MgO}$ nanofillers of different concentrations measured at room temperature are shown in Figure 3. The $\varepsilon_{\mathrm{r}}$ increases initially in the frequency range of $10-500 \mathrm{~Hz}$ and beyond $500 \mathrm{~Hz}$ it decreases with increase in frequency. This observation is related to the polarization of dipoles oscillation with change in applied field associated with each of PAEK and $\mathrm{MgO}$ nanofillers. Initially, the increase in $\varepsilon_{\mathrm{r}}$ is due to the instantaneous change in electronic polarization within the material which is in phase with the change in electric field. Above $500 \mathrm{~Hz}$, the atomic and orientational polarization of dipole groups may associate with PAEK which are responsible for the decreasing trend of $\varepsilon_{r_{r}}$ At these frequencies the orientation of different polar groups may get attached to the PAEK chain and participate in the polarization process.

For a particular frequency of the electric field, the dipole polarization mechanisms in PAEK can be deduced if the mobilities of the contributing dipolar groups which allow them to orient with the electric field are constrained. Therefore, with increase in frequency the time associated with the orientation of dipolar groups of PAEK differs with that of the applied electric field, hence there is a decrease observed in $\varepsilon_{\mathrm{r}}$. Also, due to the reduction in the electrical polarization mechanisms in the bulk of the material the effective dielectric constant decreases with increase in frequency. In a polymer composite system, the contribution of the filler polarization would result in an enhancement in the composite dielectric constant above that of the unfilled polymer. Presently, the dielectric constant variation is not too high, this may be due to the fact that the effect of the filler dielectric constant is not much influenced by the prepared PAEK nanocomposites. 

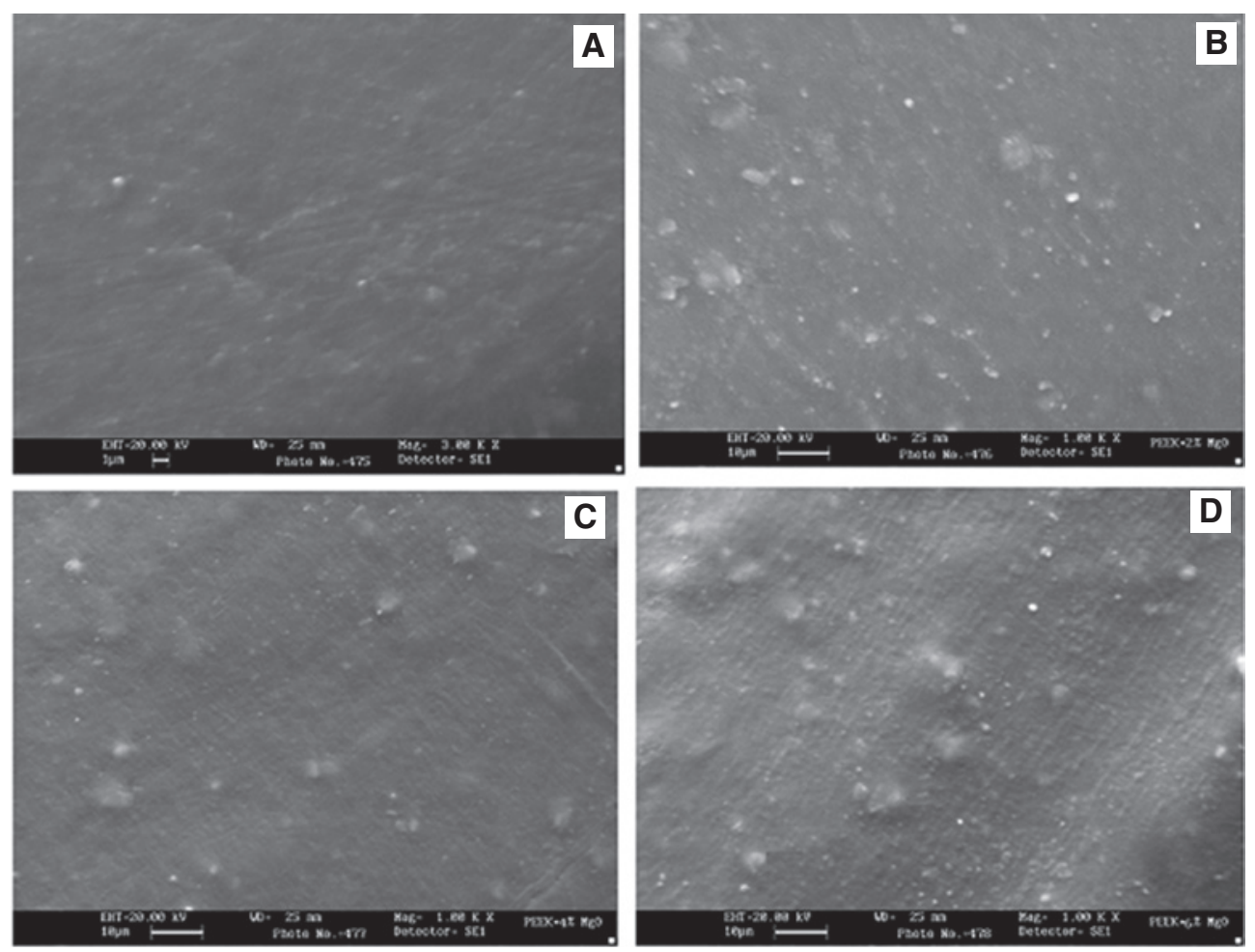

Figure 2: SEM images of PAEK-MgO nanocomposites: (A) $0 \mathrm{wt} \%$, (B) $2 \mathrm{wt} \%$, (C) $4 \mathrm{wt} \%$ and (D) $6 \mathrm{wt} \%$ of MgO nanoparticles.

\subsubsection{Effect of temperature on dielectric constant}

From Figure 4, it can be seen that the dielectric constant $\left(\varepsilon_{\mathrm{r}}\right)$ of nanocomposites almost remains constant $\sim 2.9$ irrespective of the filler loading up to $150^{\circ} \mathrm{C}$. Beyond $150^{\circ} \mathrm{C} \varepsilon_{\mathrm{r}}$ increases rapidly with an increase in temperature. The variation of $\varepsilon_{\mathrm{r}}$ may depend on several factors such as: (i) polarizability of the moieties on the chain, (ii) pendant groups hanging on the chain, (iii) orientation of the

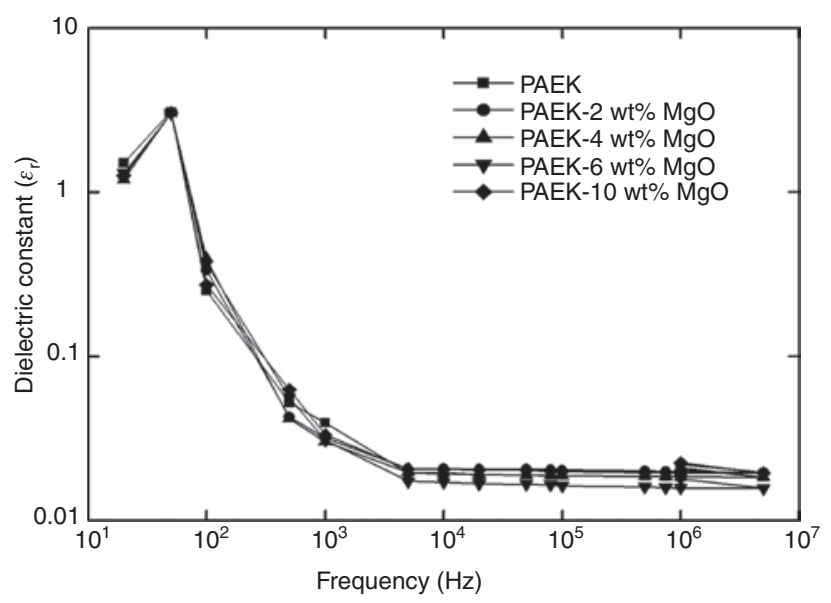

Figure 3: Effect of frequency on dielectric constant for PAEK-MgO nanocomposites. groups and their interaction with the surrounding groups, (iv) segmentation movement of polymeric chain, (vi) their synchronization with the applied frequency, and (vii) accompanied losses. The PAEKs are nonpolar in nature and have aromatic rings coupled with the ether and ketone groups which may tend to exhibit lower polarizability of the chain and stiffness of the ketone groups against the flexibility of ether linkages. Therefore, the capacitive currents arising due to the polarization of

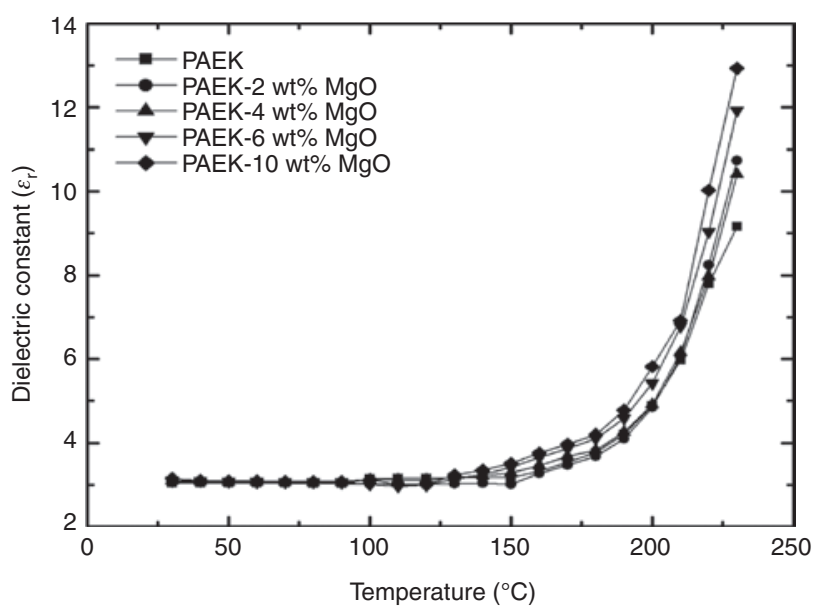

Figure 4: Effect of temperature on dielectric constant for PAEK-MgO nanocomposites. 
dipoles may be limited which holds the charges. This property may make the nanocomposites more suitable for power transformer cable insulation applications. The polymer chain as well as pendant groups of PAEK does not exhibit high polarizability at the temperature below $100^{\circ} \mathrm{C}$, hence the $\varepsilon_{\mathrm{r}}$ variation is not high. Due to the inclusion of metal oxide nanofillers into PAEK, there might be slight reduction in free volume. Beyond $100^{\circ} \mathrm{C}$ there is an increase in $\varepsilon_{\mathrm{r}}$ as shown in Figure 4, which might be due to the expanding nature of the PAEK polymer.

\subsubsection{Effect of frequency on $\tan \delta$}

The effect of frequency on $\tan \delta$ of PAEK/MgO nanocomposites at room temperature is shown in Figure 5 as a function of $\mathrm{MgO}$ nanofiller concentration. The $\mathrm{PAEK} / \mathrm{MgO}$ nanocomposites showed an increase in tan $\delta$ values with increase in frequency, but at frequencies greater than $750 \mathrm{~Hz}$ the $\tan \delta$ values decrease with an increase in frequency. At frequencies lower than $750 \mathrm{~Hz}$ $\tan \delta$ is higher in PAEK/MgO nanocomposites, which might be due to lower energy level activations of charge carriers occurring due to dipole relaxations at these frequencies. With increase in frequency above $750 \mathrm{~Hz}$ the overall rigidity of the back bone chains of PAEK and its nanocomposites may lead to lower dipolar orientation losses which might result in lower $\tan \delta$. As seen, $\tan \delta$ decreases with increasing content of $\mathrm{MgO}$ nanofillers. This effect may reduce the interaction between polymer chains and polar pendant interactions with the applied electric field. The moisture present in the nanocomposite

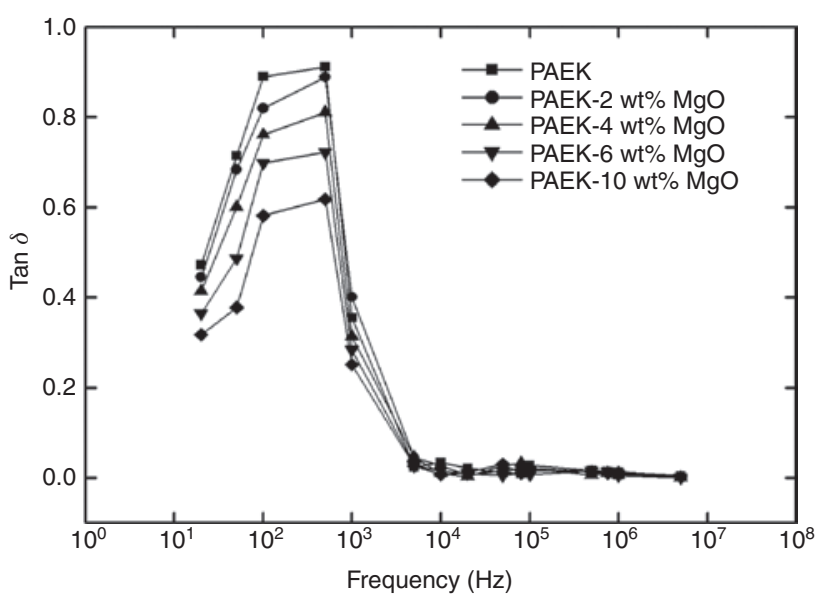

Figure 5: Effect of frequency on $\tan \delta$ for PAEK-MgO nanocomposites. samples may also contribute to higher losses in this frequency range.

\subsubsection{Effect of temperature on $\tan \delta$}

Figure 6 shows the effect of temperature on $\tan \delta$ of PAEK/ $\mathrm{MgO}$ nanocomposites. It has been observed that the $\tan \delta$ of the nanocomposites are fairly constant up to $150^{\circ} \mathrm{C}$, and then afterwards it increases with increase in temperature. The polymer PAEK and its nanocomposites have shown very low values of $\tan \delta$ ranging from 0.0001 at room temperature to 0.0025 at $150^{\circ} \mathrm{C}$. This behavior is attributed to the presence of the low polarizable component and high ketone component in PAEK molecule as compared to conventional PEEK polymer available in the market. This makes the molecular chains in PAEK more rigid and compact leading to higher melting point and higher thermal stability at these temperatures. Generally, lower $\tan \delta$ indicates the suitability of the polymeric composites for dielectric applications such as power transformer windings. Another reason for low values of $\tan \delta$ may be the nonsynchronization of the polarization process and applied oscillating electric potential within the nanocomposite system as observed by Ref. [12]. Polarization in the polymer composite is due to orientation, atomic and electronic polarization. With the application of an electric field the polarization of the molecules takes place and once the potential is reversed the depolarization process is not able to follow the change in the field which results in dielectric loss within the material.

The resulting polarization and its nonsynchronization with the changing potential, leads to dielectric losses

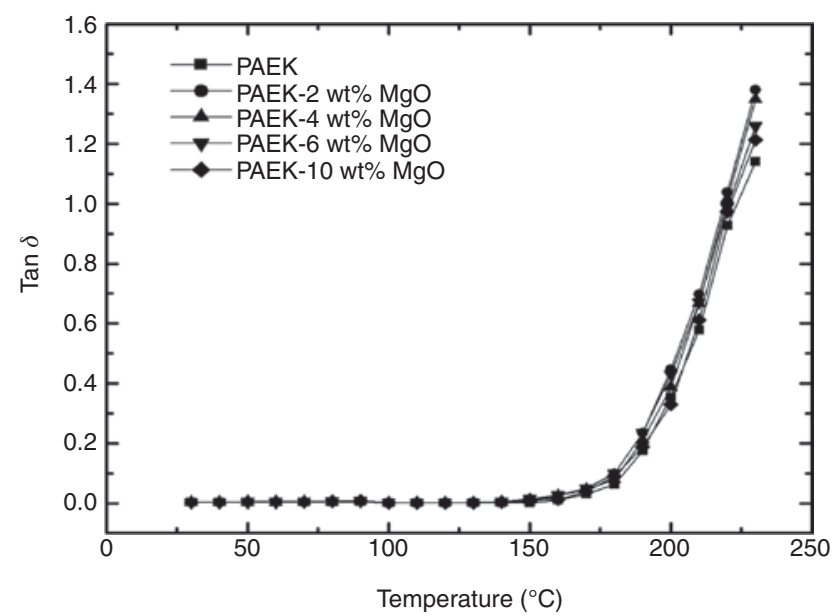

Figure 6: Effect of temperature on $\tan \delta$ for PAEK-MgO nanocomposites. 
known as dielectric relaxation. The dielectric relaxation in PAEK/MgO nanocomposites might depend on the range of energy loss mechanisms ( $\alpha, \beta$ and $\gamma$ relaxation) and temperature. The $\alpha$ relaxation is a high energy range loss mechanism which may be due to the glass transition and the polymer chain movements within the nanocomposites $[13,14]$. Beta relaxation is a medium energy range loss mechanism, may be due to conformational flips of the cyclic units in the chain and also due to rotation of polar groups around the main chain of the polymeric system while $\gamma$ relaxation is the low energy range loss whose variation may be due to the segmental motion of polymeric chains. Increase in $\tan \delta$ at temperatures above $200^{\circ} \mathrm{C}$ may be due to contaminating species like moisture, presence of polar gases due to decomposition, partial discharges of polar species in the voids and cavities, thermally excited and rheological movement of ions and species within the system.

\subsubsection{Effect of temperature on volume resistivity}

Figure 7 shows the effect of temperature on log volume resistivity $\left(\rho_{\mathrm{v}}\right)$ for PAEK/MgO nanocomposites. From the figure three distinct regions in $\rho_{\mathrm{v}}$ are observed: (i) $25^{\circ} \mathrm{C}$ to about $100^{\circ} \mathrm{C}$, (ii) about $100^{\circ} \mathrm{C}-175^{\circ} \mathrm{C}$ and (iii) about $175^{\circ} \mathrm{C}-225^{\circ} \mathrm{C}$. The obtained $\rho_{\mathrm{v}}$ for the PAEK nanocomposites is above $1 \times 10^{16}$ indicating they are more insulating in nature. This might be due to the absence of polarizable moieties in the matrix at low temperature resulting in very low leakage current flow from one end to another end of the electrode. In regions (i) and (ii) $\rho_{\mathrm{v}}$ decreases insignificantly with increasing temperature up to about $100^{\circ} \mathrm{C}$ after which it decreases significantly with increasing

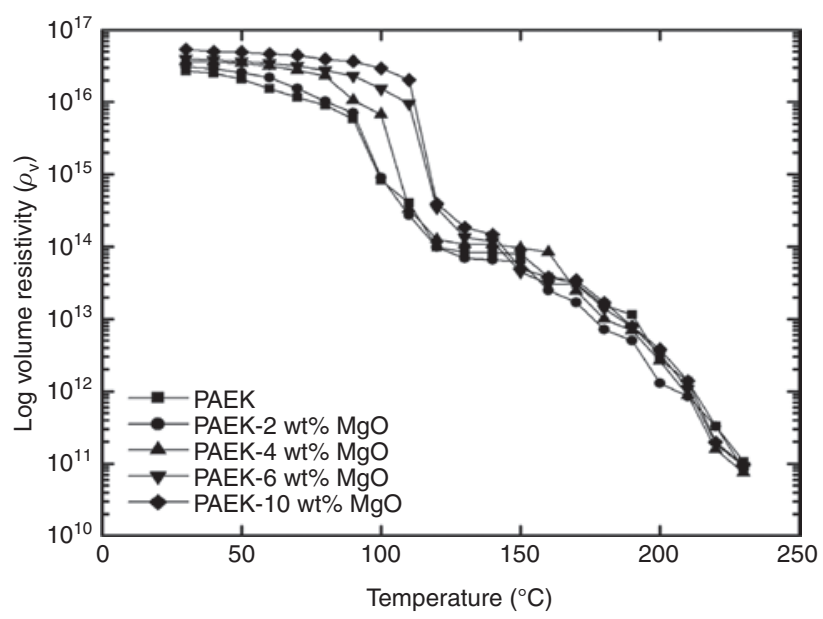

Figure 7: Effect of temperature on $\rho_{\mathrm{v}}$ for PAEK-MgO nanocomposites. temperature. This decrease in $\rho_{\mathrm{v}}$ may be due to the appearance of moisture molecules that are gaseous and polar in nature. In region (iii) the volatile and dissociation products in the nanocomposites contribute to the formation of polar moieties which generate leakage currents, thereby reducing the $\rho_{\mathrm{v}}$. In PAEK family perfect chain packing is difficult and this is manifested by maximal achievable crystallinity of $<50 \%$. The PAEK polymers are considered to have good thermal stability which disallows the formation of degradation products. Hence, PAEK/MgO nanocomposites are able to maintain high volume resistivities in the measured range of temperatures.

\subsubsection{Effect of filler loading on $\varepsilon_{\mathrm{r}}, \tan \delta$ and $\rho_{\mathrm{v}}$}

It has been observed that $\varepsilon_{\mathrm{r}}$ of the PAEK/MgO nanocomposites increases with increase in filler loading at $230^{\circ} \mathrm{C}$ as shown in Table 1. With increasing filler content, the permittivity increases due to the filler grade and permittivity of the metal oxide filler. According to the conventional mixture rules for composites, the dielectric constant of the compound should be higher than the base material. According to the interface model, the immobilization of the polymer layer determines the real permittivity of the composites with respect to effective medium or integral methods. At $4 \mathrm{wt} \%$ the immobilization of the particle surfaces diminishes the influence of the higher permittivity of the particles, thus a slight reduction in the dielectric constant of the compound results due to structural changes [15].

It has been observed that $\tan \delta$ of the PAEK/MgO nanocomposites is greater at $2 \mathrm{wt} \%$ filler loading than the plain PAEK whereas it decreases at greater contents of metal oxide nanofillers as shown in Table 1 . This might be due to the possibility of high temperature induced molecular motions in the form of conductive current, which help electronic or ionic charge carriers hopping in the trap sites at $2 \mathrm{wt} \%$ filler loading. Further, with the addition of $10 \mathrm{wt} \% \mathrm{MgO}$ nanoparticles, the particle distance is reduced which restricts the hopping mechanism in the trap sites as

Table 1: Effect of filler loading on dielectric properties of PAEK-MgO nanocomposites.

\begin{tabular}{llllll}
\hline Parameter & \multicolumn{6}{l}{ Filler loading $(\mathbf{w t} \%)$ at $\mathbf{2 3 0 ^ { \circ } \mathrm { C }}$} \\
\cline { 2 - 6 } & $\mathbf{0}$ & $\mathbf{2}$ & $\mathbf{4}$ & $\mathbf{6}$ & $\mathbf{1 0}$ \\
\hline$\varepsilon_{\mathrm{r}}$ & 9.16 & 10.73 & 10.40 & 11.93 & 13.0 \\
$\operatorname{Tan} \delta$ & 1.14 & 1.38 & 1.35 & 1.26 & 1.21 \\
$\log \rho_{\mathrm{v}}$ & $1.06 \mathrm{E} 11$ & $8.25 \mathrm{E} 10$ & $7.45 \mathrm{E} 10$ & $9.25 \mathrm{E} 10$ & $9.55 \mathrm{E} 10$ \\
\hline
\end{tabular}


compared to base polymer. The $\rho_{\mathrm{v}}$ slightly decreases with increase in filler loading as compared to PAEK. At $230^{\circ} \mathrm{C}$ the $\rho_{\mathrm{v}}$ seems to be almost equal for all the PAEK/MgO nanocomposites as observed from Table 1 . The highest value of $\rho_{\mathrm{v}}$ is attained at $10 \mathrm{wt} \%$ filler loading, which indicates low concentrations of polar contaminants within the system. The $\mathrm{MgO}$ nanoparticles developed by sol-gel technique which is in the form of compacted powders, may have more complex structure (as observed from TEM images Figure 1), and is typically an assembly of approximately equiaxed crystallites, with the limited amount of porosity as a highly dispersed continuous phase results in increase of $\rho_{\mathrm{v}}$ according to a reference paper [16].

\subsection{Differential calorimetric analysis}

The differential scanning calorimetry (DSC) heating curves corresponding to PAEK and PAEK/MgO nanocomposites are shown in Figure 8. In DSC heating curves, two distinct thermal zones are observed. The first zone is in the region near $300^{\circ} \mathrm{C}$ where the polymer starts to soften at the onset melting temperature $\left(T_{\mathrm{m}}\right.$ on $)$, and the polymer completely melts at $350^{\circ} \mathrm{C}$ and is designated as the melting temperature $\left(T_{\mathrm{m}}\right)$. It was observed that the peak value, $T_{\mathrm{m}}$ is slightly deviated with the addition of $\mathrm{MgO}$ nanoparticles. Existing literature on the subject indicates that the thermal properties of PEEK polymer composites largely depend upon the type of fillers, processing conditions, and matrix-filler interactions $[8,17,18]$. In the present study, even though there is no significant change in the $T_{\mathrm{m}}$ values, drastic change in the $T_{\mathrm{m}}{ }^{\text {on }}$ was observed with increase in the filler loading of $\mathrm{MgO}$. In pure PAEK polymer, the melting starts around $325^{\circ} \mathrm{C}$ whereas, for the PAEK/MgO nanocomposites with $2 \mathrm{wt} \%$ filler loading

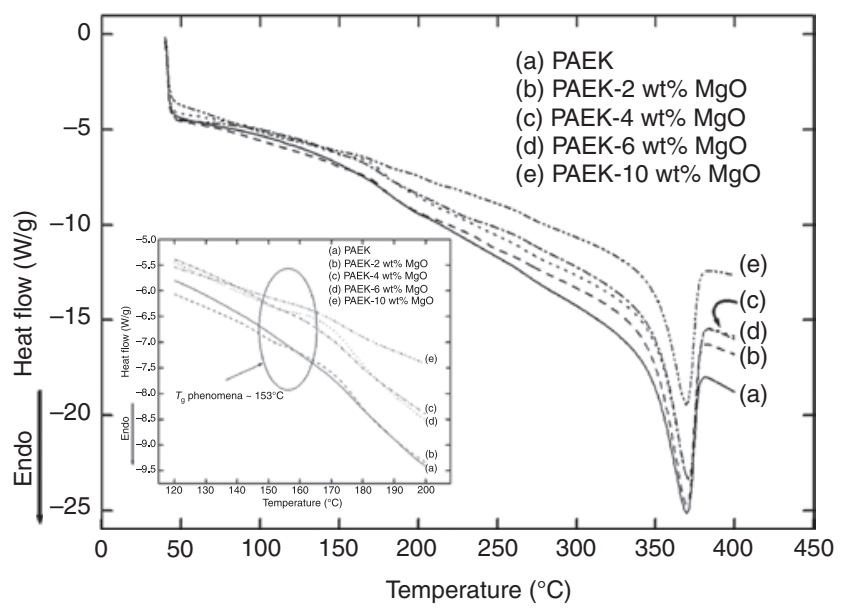

Figure 8: DSC plot of PAEK-MgO nanocomposites. melting starts around $350^{\circ} \mathrm{C}$. The melting temperature does not change unless a polymer is chemically modified at the molecular level [19]. In the present study, not much difference is observed in the melting temperature of PAEK/MgO nanocomposites, this might be due to the physical interaction of nanofiller phase with PAEK. Because of this, the molecular nature of the polymer was unaffected. However, $\mathrm{MgO}$ nanofillers can effectively retard the segmental movement of PAEK polymer chains thereby increasing the onset melting temperature of the composites. Therefore, the semicrystalline aromatic polymers such as PAEK can maintain good strength even at high temperatures.

\subsection{Thermo-mechanical analysis}

Dimensional stability of the composite material at high temperature regions is an important characteristic of the dielectric material during its service in the power equipment. Figure 9A,B shows the plot of thermo-mechanical

A
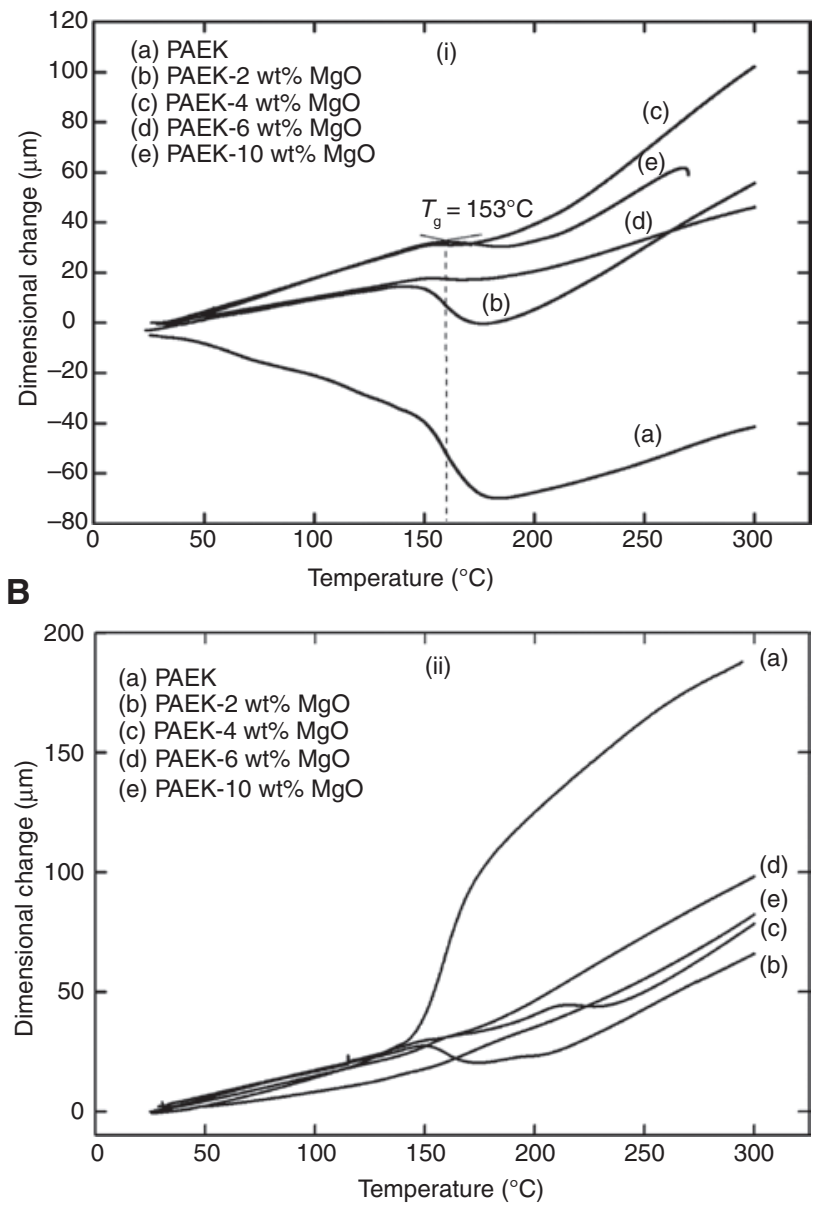

Figure 9: TMA plots using (A) penetration probe and (B) expansion probe for PAEK-MgO nanocomposites. 
analysis (TMA) for PAEK and PAEK/MgO nanocomposites. The penetration probe provides data on the softening properties of thermoplastics. This probe is placed at the geometric center of the top portion of the square sample. Due to the influence of the heat and the applied force on the penetration probe, the sample sags uniformly without diminishing in its sectional thickness. As opposed to PAEK/MgO nanocomposites, the PAEK sample shows appreciable penetration of the probe indicating material softening.

It is important that, at high temperatures, penetration into the material indicates how well the material has softened and sticks to the conductor. High penetration indicates that the material is softening and a dimensional change has taken place. From Figure 8, we can observe that $T_{\mathrm{g}}$ of the PAEK and PAEK/MgO nanocomposites is $\sim 153^{\circ} \mathrm{C}$ the variation in the penetration probe senses the dimensional change of material and returns to the normal conditions. With further increase in temperature above $T_{\mathrm{g}}$, the PAEK/MgO nanocomposites have maintained their dimensional stability with variation in the force applied by the penetration probe. This behavior in nanocomposite is due to the filling effect of $\mathrm{MgO}$ which provides the dimensional compactness at $T_{\mathrm{g}}$ and beyond $T_{\mathrm{g}}$. From Figure 9A it is also observed that the effects of the dimensional stability using a penetration probe has a much smaller contact diameter and is able to penetrate more, hence the softening is much pronounced in these plots. Figure 9B shows the TMA plot for PAEK and $\mathrm{PAEK} / \mathrm{MgO}$ nanocomposites using the expansion probe method. In this method, the probe rests on the surface of the test specimen under low loadings. Initially, as the temperature increases from room temperature, the sample expands and the probe is pushed up. This results in the expansion of the sample which can be measured. In this method also, the observed $T_{\mathrm{g}}$ is $\sim 153^{\circ} \mathrm{C}$. At $T_{\mathrm{g}}$, the PAEK matrix exhibits a significant change in slope due to an increase in its rate of expansion, whereas the rate of expansion is lower for PAEK/MgO nanocomposites due to the filling effect of the $\mathrm{MgO}$ nanofiller. The onset temperature of this change in expansion behavior is due to the $T_{\mathrm{g}}$ of PAEK nanocomposites. On the other hand, voids are typically not desired in the polymer composites used for dielectric applications. They create a suitable space for allowing moisture, air and other polar contaminants in the material. Under an applied electric potential the presence of voids leads to partial discharges, increases the dielectric loss, and degrades the dielectric leading to ultimate failure. Hence, it would be desirable to have the polymer composites compact and devoid of air gaps so that they can perform well as dielectrics. In the present study, a high degree of packing devoid of pores would have facilitated the reduction of the softening of composite at the tested temperatures.

\subsection{Thermal conductivity}

Normally polymers are poor conductors of heat. In order to improve the thermal conductivity $(\gamma)$ of PAEK, $\mathrm{MgO}$ nanoparticles were selected whose $\gamma$ was $38 \mathrm{~W} / \mathrm{mK}$ [20], thereby enhancing the overall $\gamma$ of the nanocomposite. The $\gamma$ values of the PAEK/MgO nanocomposites are listed in Table 2. From Table 2, it was observed that, $\gamma$ and diffusivity have been increased with increase in $\mathrm{MgO}$ loading and the improvement of $\gamma$ for the studied range of $\mathrm{MgO}$ content is about $12.7 \%$. Thermal conductivity is a kinetic property determined by the contributions from the vibration, rotational and electronic degrees of freedom. In the tested range of temperature range $300^{\circ} \mathrm{C}$, the conduction in $\mathrm{MgO}$ is essentially considered as ionic in nature [16], which depends on magnesium cation vacancies, lower activation energy during the conduction process, and crystalline surfaces. As $\mathrm{MgO}$ nanofillers are considered as high temperature insulators heat transfer within the composite system occurs at a higher rate due to increased $\gamma$. From the solid state theory, an increase in temperature causes an increase in the intermolecular vibrations, which is a dominant mechanism for heat transfer. In the present study, $10 \mathrm{wt} \%$ nanocomposites has high value of thermal conductivity which means that heat travels easily through the material. With the increase in filler loading thermal diffusivity increases by $16 \%$ and is maximum at $10 \mathrm{wt} \%$ nanofiller loading. This signifies that at $10 \mathrm{wt} \%$ nanocomposite will experience a quick rise in the temperature. In contrast, pure PAEK exhibits a high value of specific heat, thus it requires more heat to raise the temperature of the material, but with the increase in filler loading this effect slightly decreases.

Table 2: Thermal conductivity of PAEK-MgO nanocomposites.

\begin{tabular}{lrrr}
\hline Sample & $\begin{array}{r}\text { Thermal } \\
\text { conductivity } \\
(\boldsymbol{\gamma})(\mathbf{W} / \mathrm{mK}) \\
(\mathbf{\pm 0 . 1 1 )}\end{array}$ & $\begin{array}{r}\text { Diffusivity } \\
\left(\mathrm{mm}^{2} / \mathbf{s}\right) \\
(\mathbf{\pm 0 . 0 1 )})\end{array}$ & $\begin{array}{r}\text { Heat } \\
\text { capacity } \\
\left(\mathbf{M J} / \mathbf{m}^{3} \mathbf{K}\right) \\
(\mathbf{\pm 0 . 1 3 )}\end{array}$ \\
\hline Neat PAEK & 0.30825 & 0.2464 & 1.251 \\
PAEK-2 wt\% MgO & 0.30941 & 0.2629 & 1.177 \\
PAEK-4 wt\% MgO & 0.31095 & 0.2665 & 1.167 \\
PAEK-6 wt\% MgO & 0.32137 & 0.2697 & 1.192 \\
PAEK-10 wt\% MgO & 0.34744 & 0.2860 & 1.215 \\
\hline
\end{tabular}




\subsection{Coefficient of thermal expansion}

The CTE values of PAEK/MgO nanocomposites are listed in Table 3. The CTE is a quantitative assessment of the expansion of a material over a temperature interval. In designing a structural material, dimensional stability with temperature is a very crucial factor. From Table 3, it is observed that the CTE was reduced by the addition of $\mathrm{MgO}$ nanofillers after $T_{\mathrm{g}}$, i.e. between temperature $250^{\circ} \mathrm{C}$ and $260^{\circ} \mathrm{C}$ as compared to pure PAEK. The CTE is almost constant with the addition of $\mathrm{MgO}$ nanofillers when the temperature is above and below $T_{\mathrm{g}}$. The reason for higher CTE above $T_{\mathrm{g}}$ as compared to below $T_{\mathrm{g}}$ may be due to the greater segmental mobility of PAEK polymer chains in the amorphous domains.

Lowering of CTE may be due to several factors: (i) reduction in free volume, which reduces the expansion of PAEK polymer, (ii) hindrance of segmental movement of polymer chains within the composite material, and (iii) increase in volume of the polymer chains. Several other researchers have shown that, in ceramic polymer composites the ceramic particles are surrounded by tightly bound (constrained) and loosely bound (unconstrained) polymer chains. With the addition of more reinforcement particles, unconstrained polymer chains get converted to constrained chains as a result of reduction in the interparticle distance. This effect retards the thermal expansion of the polymer composites $[8,21]$. In the present study, during composite fabrication, the $\mathrm{MgO}$ nanoparticles might be randomly aligned in both lateral and horizontal directions within the PAEK matrix which prevents the expansion of PAEK matrix. It was reported that the bulk CTE of $\mathrm{MgO}$ is only $13.5^{\circ} \mathrm{C}$ [19], which is much less than the pure PAEK. This may also be a probable reason for the reduction in CTE upon $\mathrm{MgO}$ nanoparticles inclusion. Nanoparticles provide a larger surface area than conventional microparticles and hence are more effective in reducing the CTE of polymer matrices [22].

Table 3: Coefficient of thermal expansion (CTE) of PAEK-MgO nanocomposites.

\begin{tabular}{|c|c|c|}
\hline Sample & $\begin{array}{r}\mathrm{CTE}\left(\mu \mathrm{m} /{ }^{\circ} \mathrm{C} \pm 0.11\right) \\
\text { (between } 50^{\circ} \mathrm{C} \text { and } \\
\left.60^{\circ} \mathrm{C}\right)\end{array}$ & $\begin{array}{r}\mathrm{CTE}\left(\mu \mathrm{m} /{ }^{\circ} \mathrm{C} \pm 0.11\right) \\
\left(\text { between } 250^{\circ} \mathrm{C}\right. \\
\left.\text { and } 260^{\circ} \mathrm{C}\right)\end{array}$ \\
\hline PAEK & 0.203 & 0.658 \\
\hline PAEK- 2 wt $\%$ MgO & 0.221 & 0.498 \\
\hline PAEK-4 wt $\%$ MgO & 0.245 & 0.490 \\
\hline PAEK-6 wt\% MgO & 0.201 & 0.495 \\
\hline PAEK-10 wt $\%$ MgO & 0.180 & 0.487 \\
\hline
\end{tabular}

\section{Conclusions}

Based on the present results and discussion the following conclusions can be drawn:

1. The PAEK/MgO nanocomposites were prepared successfully via extrusion followed by injection molding. The TEM micrographs indicate that PAEK/MgO nanocomposites show uniform dispersion of $\mathrm{MgO}$ in PAEK matrix material.

2. The dielectric constant and $\tan \delta$ of PAEK and its nanocomposites reduce with increase in frequency. At a maximum applied temperature of $230^{\circ} \mathrm{C}$, a lower $\tan \delta$ of 1.2 and higher dielectric constant of 13 were obtained at $10 \mathrm{wt} \% \mathrm{MgO}$ filled nanocomposite.

3. The volume resistivity is observed to be maximum at $10 \mathrm{wt} \% \mathrm{MgO}$ filled nanocomposite but it is lower than that of the neat PAEK material.

4. Further, highest thermal conductivity, high diffusivity and lower coefficient of thermal expansion were also obtained at $10 \mathrm{wt} \%$ filler loading.

5. The resulting PAEK/MgO nanocomposites exhibited excellent thermal properties, with a high glass transition temperature of $153^{\circ} \mathrm{C}$, which might be due to the complex structure or three-dimensional nature of $\mathrm{MgO}$ nanofillers restricting the mobility of the polymeric chains resulting in less $T_{\mathrm{g}}$ variation.

6. Within the studied range of $\mathrm{MgO}$ content $0-10 \mathrm{wt} \%$ we conclude that from the novel method of fabricated PAEK/MgO nanocomposites $10 \mathrm{wt} \% \mathrm{MgO}$ nanofiller reinforcement seems to be the optimum loading to produce improved performance in the PAEK/MgO filled nanocomposites which can be used for tailormade applications and specifically as insulators for power equipments.

7. When the loading of $\mathrm{MgO}$ nanoparticles is above $10 \mathrm{wt} \%$ there was inhomogeneous dispersion of nanoparticles in the matrix with localized domains and phase segregation at the nanometer level. Therefore, the fabrication of nanocomposites above $10 \mathrm{wt} \%$ loading has not shown good results for the experimental work, hence the results were concluded up to this loading.

Acknowledgments: The support and encouragement of the management of Central Power Research Institute (CPRI, Bengaluru, India) for providing all the infrastructure, financial, and encouragement for carrying out this research activity is greatly acknowledged. Thanks to JSS Research Foundation (JSSRF, Sri Jayachamarajendra College of Engineering, Mysuru, India) for all the necessary 
infrastructural support. The authors also thank both the managements for the kind help and support rendered towards the project.

\section{References}

[1] McNutt WJ, Provost RL, Whearty RJ. IEEE Trans. Power Delivery 1999, 11, 1391-1399.

[2] Xu Y, Qitong W, Yunhe Z, Zhenhua J. Polym. J. 2012, 44, 1042-1047.

[3] Juan X, Wei-Ya P, Guang L, Jian-MJ. J. Fiber Bioeng. Inf. 2010, 3, 142-147.

[4] Vaishnav D, Goyal RK. 2nd Int. Conf. Struct. Nano Compos. IOP Conf. Series Mater. Sci. Eng. 2014, 64, 1-10, 012016. doi:10.1088/1757-899X/64/1/012016.

[5] Geng Z, Huo M, Mu J, Zhang H, Lu Y, Luan J, Huo P, Du Y, Wang G. J. Mater. Chem. C 2014, 2, 1094-1103.

[6] Goyal RK, Kapadia AS. Compos. Part B 2013, 50, 135-143.

[7] Goyal RK, Tiwari AN, Mulik UP, Negi YS. Comp. Sci. Technol. 2007, 67, 1802-1812.

[8] Goyal RK, Tiwari AN, Mulik UP, Negi YS. Compos. Part A 2007, 38, 516-524.

[9] Di Vona ML, Sgreccia E, Donnadio A, Casciola M, Chailan JF, Auer G, Knauth P. J. Mem. Sci. 2011, 369, 536-544.
[10] Mishra TK, Ashish K, Vishal V, Pandey KN, Vijai K. Comp. Sci. Technol. 2012, 72, 1627-1631.

[11] Liu T, Li Y, Su Y, Yu H, Zhao N, Yang Y, Jiang Z. Polym. Compos. 2016, 37, 3003-3014.

[12] Blythe T, Bloor D. Electrical Properties of Polymers, Cambridge University Press: New York, 2005.

[13] Navriliak S, Havriliak SJ. Dielectric and Mechanical Relaxation in Materials - Analysis, Interpretation and Application to Polymers, Hanser Pub: Munich, 1997.

[14] Ahmad Z. Polymeric Dielectric Materials, Intech Europe, 2012.

[15] Andritsch T, Kochetov R, Morshuis PHF, Smit JJ. DC conduction in epoxy based nano- and mesocomposites At 2010 Annual Report Conference on Electrical Insulation and Dielectric Phenomena (CEIDP), USA, 17-20 Oct 2010, pp. 1-4. doi:10.1109/ CEIDP.2010.5723965.

[16] Wilson IO. IEE Proc. 1981, 128, 159-164.

[17] Kuo MC, Tsai CM, Huang JC, Chen M. Mater. Chem. Phys. 2005, 90, 185-195.

[18] Goyal RK, Negi YS, Tiwari AN. J. Appl. Polym. Sci. 2006, 100, 4623-4631.

[19] Mahesh KV, Balanand S, Raimond R, Peer Mohamed A, Ananthakumar S. Mater. Design 2014, 63, 360-367.

[20] Callister WD. Materials Science and Engineering, 7th ed., John Wiley \& Sons Inc.: New York, 2007.

[21] Tsagaropoulos G, Eisenberg A. Macromolecules 1995, 28, 6067-6077.

[22] Rong MZ, Zhang MQ, Zheng YX, Zeng HM, Friedrich K. Polymers 2001, 42, 3301-3304. 